\title{
Ueber Trematoden
}

\section{der \\ Süsswasserfische}

von

\section{Leopold HAUSIMA N N}

in Basel

Hierzu Tafel 1

Vorliegende Arbeit beschäftigte mich vom Mai 1895 bis Juli 1896 im zoologischen Institut der Universität Basel.

Es ist mir eine angenehme Pflicht meinem hochverehrten Lehrer Herrn Prof. Dr. Fr. ZschoKke für die Anregung mich auf diesem Gebiete zu bethätigen, für das rege Interesse, welches er meiner Arbeit entgegenbrachte, sowie für den bewährten Rat, mit dem er mir stets zur Seite stand, meinen wärmsten Dank auszusprechen.

Auch dem früheren Assistenten Herrn Dr. O. Fuhrmann, der mich in technischer Beziehung in die Helminthologie einführte, fühle ich mich zu grossem Dank verpflichtet. 


\section{I.}

\section{Fannistisch-biologischer Teil.}

\section{A. Binleitende Bemerkingen.}

Erst in den letzten Jahrzehnten sind die Parasiten der Süsswasserfische in faunistischer Beziehung von ZscноккE (1884), Piesbergen (1886), Prenant (1886) u. a. einem eingehenderen Studium unterworfen worden.

Bei Aufnahme der vorliegenden Arbeit war es mir ebenfalls besonders darum zu thun die Parasitenfauna der Fische, speciell der Fische aus Basels Umgebung, genauer kennen zu lernen.

Es handelt desshalb der erste Teil der Arbeit hauptsächlich von den Verhältnissen, welche sich für das Vorkommen entoparasitischer Trematoden in Fischen geltend machen können. Zum Zwecke deutlicher Uebersicht wurden statistische Tabellen beigegeben.

Der zweite Teil wird eine eingehendere, anatomisch-histologische Untersuchung einer neuen Species und einer neuen Varietät geben. Ebenso finden sich in demselben verschiedene Beobachtungen aufgezeichnet, die ich noch an anderen von mir untersuchten Trematoden gemacht habe.

Bevor ich zur eigentlichen Ausführung der Arbeit übergehe, mögen noch einige Bemerkungen über das Gebiet, die Methoden und die Objekte der Untersuchung Platz finden.

\section{a) Das Untersuchungsgebiet.}

Die von mir untersuchten Fische erwarb ich zum grössten Teil lebend, indem ich dieselben von einigen Fischern Basels und der Umgebung bezog. Es entstammen die meisten dem 
Rhein. Sie wurden zum Teil bei Basel, zum Teil bei Istein und Mumpf - letzterer Ort liegt in der Nähe von Rheinfelden gefangen.

Es war mir ferner ermöglicht, viele Eingeweide von Fischen zu untersuchen, deren Beschaffung ihrer Körpergrösse wegen $\mathrm{zu}$ schwierig gewesen wäre.

Die Untersuchung wurde auch im letzteren Falle nur an frischem Material unternommen.

Aus der Menge der Parasiten, welche dabei zu Tage gefördert wurden, habe ich nur die Trematoden herausgegriffen. Die Resultate, welche ich zu gleicher Zeit an anderen Parasiten der Fische des angegebenen Untersuchungsgebietes erzielt habe, hoffe ich später veröffentlichen zu können.

\section{b) Untersuchungsmethoden.}

Das lebende Material wurde in konzentrirter wässeriger Sublimatlösung, in Pikrinessigsäure oder in Sublimatessigsäure fixirt. Mit letzterer Lösung erzielte ich besonders günstige Resultate.

Nach Durchbehandlung in der aufsteigenden Alkoholreihe ist für Färbungen von Totalpräparaten nach meinen Erfahrungen das Alaunkarmin dem Pikrokarmin vorzuziehen, jedoch fand ich letzteres für Durchfärbung von Cysten sehr günstig. Es müssen dieselben jedoch einige Tage in der stark mit destillirtem Wasser verdünnten Farbe gelassen werden.

Für Schnittpräparate wendete ich die bekannten Methoden an, wobei ich fand, dass, für meine kleinen Objekte wenigstens, das Hæmalaun nach MEYER ein vorzügliches Färbmittel sei.

\section{c) Untersuchungsobjekte.}

Die Fischfauna Basels setzt sich nach LeUthner (1877) aus 38 Arten zusammen. Nach Fatio (1882) dagegen beherbergt der Rhein nebst seinen Zuflüssen bei Basel 34 Fischspecies, 
von denen Acipenser sturio, Petromyzon marinus und Silurus glanis nur zufällig vorkommen.

Von diesen Fischen habe ich nun untersucht: Petromyzon fluviatilis; Anguilla vulgaris; Alosa vulgaris; Esox lucius; Trutta salar, lacustris, fario; Salmo salvelinus ; Thymallus vulgaris ; Cobitis barbatula; Chondrostoma nasus; Phoxinus laevis; Squalius cephalus, leuciscus, Agassizii; Scardinius erythrophthalmus; Alburnus lucidus; Blicca björkna; Abramis brama; Gobio fluviatilis ; Barbus fluviatilis ; Tinca vulgaris ; Cyprinus carpio; Lota vulgaris; Cottus gobio; Gasterosteus gymnurus; Perca fluviatilis; Acerina cernua und Lucioperca sandra.

Von den angeführten 29 Arten untersuchte ich 1029 Exemplare. Da ich aber die Beobachtung nicht auf alle Arten in gleich eingehender Weise ausdehnen konnte, so wählte ich aus den verschiedenen Gruppen einzelne Repräsentanten aus, denen ich dann eine besondere Aufmerksamkeit schenkte.

Die Distomen, denn diese sind die zahlreichsten Vertreter unter den von mir aufgefundenen Fischparasiten, sind folgende: Distomum angusticolle, exspinosum, globiporum, farionis, nodulosum, isoporum, perlatum, tereticolle, appendiculatum, ocreatum, reflexum, varicum, ventricosum.

Die übrigen Trematoden, welche sich in den vorhin angeführten Wirten noch vorfanden, sind: Gasterostomum fimbriatum, Diplozoon paradoxum, Octocotyle lanceolatum, Tetraonchus unguiculatus, Tetracotyle spec.?

Ausser diesen fand ich noch einige andere Trematoden, von denen es mir aus verschiedenen Gründen unmöglich war, Diagnosen oder Bestimmungen geben zu können. 


\section{B. Der Einfluss der Lebensweise des Wirtes anf seine Parasiten.}

\section{a) Der Einfluss der Nahrung des Wirtes auf seine Schmarotzer.}

Allgemein nimmt man gegenwärtig an, dass die Uebertragung der Larvenstadien der digenetischen Trematoden auf zweierlei Art erfolgen kann. Entweder wandern dieselben in den neuen Wirt aktiv ein, oder gelangen mit dem Wirt passiv auf den Hauptwirt, indem ersterer von letzterem aufgefressen wird. Die passive Uebertragung ist in der Regel die vorherrschende.

Da nun die Süsswasserfische vielfach die Endwirte der Distomen, denn diese kommen bei meinen Beobachtungen besonders in Betracht, sind, so ist es von Interesse, den Einfluss der Nahrungsverhältnisse der Fische auf besagte Schmarotzer näher zu untersuchen, denn sobald wir wissen wie und von was sich ein Tier ernährt, so können wir auch vermuten, welche Parasiten es beherbergen kann.

Die Nahrung der Fische ist bei verschiedenen Gruppen verschieden. Man hat die Fische desshalb in dieser Hinsicht eingeteilt in Carnivoren, Omnivoren und Herbivoren. Susta (1888), der zum Zwecke der Fischzucht eingehende Studien über die Fischnahrung gemacht hat, unterscheidet Raubfische oder Fischfresser, Kleintierfresser und Grünweidefische oder Pflanzenfresser.

Nun finden sich aber die verschiedensten Angaben über die Ernährungsweise ein und desselben Fisches, so dass es oft schwer hält das Richtige zu finden. Ja oft sind diese soweit reichenden Widersprüche in den Angaben, wie SusTa (1888) erklärt, einfach nicht zu lösen. 
Nach meinen Untersuchungen, die sich ja hauptsächlich auf die Parasitenfauna beziehen, wäre es gewagt die untersuchten Fische nach ihrer Nahrung einzuteilen. Ich gebe hier desshalb eine Liste der von mir untersuchten Fische, welche der Einteilung, die SUSTA (1888) gibt, folgen wird :

Raubfische

Esox lucius L.

Trutta fario L.

Lota vulgaris Cuv. Perca fluviatilis L. Acerinu cernua L. Lucioperca sandra Cuv. Salmo salvelinus L.
Kleintierfresser

Thymallus vulgaris Nils. Squalius cephalus L.

Cobitis barbatula L.

Chondrostoma nasus L. Scardinius erythrophthal-

Phoxinus lævis Ag.

Squalius Agassizii Heck.

Alburnus lucidus Heck.

Blicca björkna L.

Abramis brama L.

Gobio fluviatilis Cuv.

Barbus fluviatilis Ag.

Tinca vulgaris Cuv.

Cyprinus carpio L.

Cottus gobio L.

Gasterosteus gymnurus Cuv.

Dass die Fische aller drei Nahrungsklassen mit Parasiten, speciell Distomen inficirt werden können, zeigt folgende Wirtstabelle :

\section{Parasit}

Distomum angusticolle exspinosum globiporum

farionis nodulosum isoporum perlatum tereticolle

appendiculatum Trutta salar. ocreatum reflexum varicum ventricosum

()
Wirt

Cottus gobio.

Barbus fluviatilis.

Chondrostoma nasus; Barbus fluviatilis; Squalius cephalus, Ieuciscus ; Cobitis barbatula; Scardinius erythrophthalmus.

Thymallus vulgaris.

Perca fluviatilis.

Barbus tluviatilis.

Tinca vulgaris; Barbus fluviatilis.

Esox lucius; Thymallus vulgaris.

Alosa vulgaris.

Gasterostomum fimbriatum Lucioperca sandra . 
Diplozoon paradoxum

Octocotyle lanceolatum Tetraonchus unguiculatus Tetracotyle spec.?
Abramis brama, Cottus gobio, Gobio fluviatilis, Phoxinus lævis.

Alosa vulgaris.

Lucioperca sandra.

Cobitis barbatula.

Die Verteilung der Parasiten auf alle drei Nahrungsklassen ihrer Wirte hat ZscHOKKe (1884) bei seinen Untersuchungen der Fische des Genfersee's schon bemerkt, wenn er schreibt: "Les Trématodes sont répartis assez régulièrement dans les différentes espèces des poissons. » Es ist dies auch leicht erklärlich, denn die Uebertragung der Schmarotzerlarven kann auf alle möglichen Wege geschehen. Inficirt kann also der pflanzenfressende Fisch, sowie der kleintier- und fleischfressende Fisch werden.

Betrachten wir dagegen den Grad der Infection, hauptsächlich aber das Stadium, in welchem die entoparasitischen Trematoden angetroffen werden, so zeigen sich für die drei Gruppen deutliche Unterschiede.

Von der Abteilung der Raubfische und Kleintierfresser hat die Untersuchung verschiedener Species negative Resultate zu Tage gefördert. -- Diejenigen Raubfische dagegen, welche von Trematoden behaftet waren, beherbergten nur geschlechtsreife Formen. Die Zahl, in welcher dieselben einen Wirt heimsuchen, ist eine ziemlich hohe.

Die Gruppe der Kleintierfresser lieferte bei meinen Untersuchungen verschiedene geschlechtsreife Trematodenarten und ein Larvenstadium. Die meisten Wirtsarten bargen Trematoden, allein es herrschte grosse Variabilität in der Menge ihres Vorkommens.

Die Grünweidefische waren ebenfalls behaftet mit Trematoden, jedoch waren dies fast durchwegs noch nicht geschlechtsreife Individuen und ebenso war die Zahl ihres Auftretens gering.

In Gegensatz zu diesen letzteren Befunden fällt es auf, dass 
die Raubfische nur entwickelte Trematoden beherbergen. Diese Thatsache ist aber in der Natur der Nahrung begründet, indem die Raubfische meistens solche Fische verzehren, deren Trematoden noch Larven sind und erst in den Räubern ihre vollständige Entwicklung erreichen. So nährt sich z. B. Perca flwviatilis, der Endwirt für Distomum nodulosum Zed., von verschiedenen Cyprinusarten, welche Zwischenwirte für diese Schmarotzerart sind.

Die Kleintierfresser, bis jetzt meist als Omnivoren bezeichnet, können, wie wir gesehen haben, sowohl von entwickelten als unentwickelten Trematoden bewohnt sein. Ohne Zweifel lässt sich das aus der Mannigfaltigkeit ihrer Nahrung erklären.

Die Pflanzenfresser sind fast nur von Larvenstadien der Trematoden bewohnt und auch diese sind wenig zahlreich. Infolge der von den übrigen Nahrungsweisen abweichenden Ernährung mit pflanzlicher Kost ist es wahrscheinlich, dass die Trematoden, welche in einen Grünweidefisch gelangen, nicht die Bedingungen finden, welche sie zu ihrer vollständigen Entwicklung benötigen. Sie gelangen infolge dessen nicht zur Eiproduction. Dies fand ich z. B. für Distomum glnbiporum Rud. aus Squalius cephalus, Squalius leuciscus und Scardinius erythrophthalmus. Es zeigten die Distomen nicht nur geringere Körpergrösse als in andern Wirten, auch die Eibildung war fast nie eingetreten. Nur ein einziges Mal fand ich einen geschlechtsreifen $d$. h. fortpflanzungsfähigen Wurm.

Es darf damit keineswegs angenommen werden, dass die genannten Fische nicht zu den Wirten des Distomum globiporum Rud. gezählt werden sollen. Dies würde durch den regelmässigen Befund widerlegt, denn ich traf besagtes Distomum im Juni und im September in Squalius cephalus; ebenso findet sich in der Liste, welche ZschokKE (1884) aufstellt, diese Species für den gleichen Wirt im Juni und August verzeichnet.

Aus diesen Erörterungen geht also folgendes hervor : 
Alle Fische, seien sie nun Pflanzen- oder Tierfresser, können den Trematoden als Wirt dienen. Der Einfluss der Nahrung des Wirtes auf seine Schmarotzer macht sich jedoch dadurch geltend, dass Raubfische nur geschlechtsreife, Pflanzenfresser meist nur unentwickelte, Kleintierfresser dagegen sowohl junge als erwachsene Trematoden beherbergen.

\section{b) Der Einfluss der Temperatur auf Wirt und Schmarotzer.}

Die Mehrzahl der Parasiten wird dem Wirte durch die Nahrung zugeführt. Das Nahrungsbedürfniss aber ist bei der Gesamtheit der Süsswasserfische von der Jahreszeit abhängig. Es fragt sich desshalb, in welcher Weise die Jahreszeiten bezw. die Temperaturverhältnisse bei der Infektion der Fische mit Trematoden mitspielen.

Indem ich meine Beobachtungen ausführe, verweise ich vorerst auf die dem Ende des faunistisch-biologischen Teiles angefügte Tabelle, in welcher ich meine Befunde nach den Monaten eingetragen habe. Ein Blick auf dieselbe genügt um zu zeigen, dass die Artenzahl der Parasiten in den Sommermonaten, von Ende Juni bis Anfang September, bedeutend grösser ist als in den übrigen, kälteren Monaten. Für einzelne Fischarten zeigt sich allerdings in den heissesten Monaten auch eine Artenreduktion ihrer Parasiten. Das verminderte Auftreten von Trematoden in der kälteren Jahreszeitl ässt, wie dies schon gethan worden ist, untrüglich darauf schliessen, dass die Temperatur nicht nur auf den Wirt sondern auch auf dessen Schmarotzer einen Einfluss ausübt.

SELIGO (1891) berichtet uns, dass das Nahrungsbedürfniss der Fische abhängig ist von der Wasserwärme. "In kaltem Wasser können die Fische wochenlang, ja manche Arten monatelang ohne Nahrung bestehen. Viele Cypriniden nehmen im Winter auch im Freien keine Nahrung zu sich; der Karpfen 
z. B. frisst nur, wenn das Wasser mindestens $9^{\circ} \mathrm{C}$. Wärme hat. Bei der Bachforelle und anderen Raubfischen nimmt zwar die Fresslust im Winter ab, hört aber nicht völlig auf. Die Forelle lässt auch im Sommer in der Ernährung nach, wenn die Wassertemperatur tiber $25^{\circ} \mathrm{C}$. steigt. »

Diese Schilderung der Temperatureinflüsse auf die Ernährung der Fische deckt sich zum grössten Teil mit dem, was ich vom parasitologischen Standpunkt aus zu schliessen vermag. Einige Beispiele mögen dies noch deutlicher zeigen. Von 137 Barben, die ich in 7 Monaten untersuchte, lieferten die Exemplare von 6 Monaten Trematoden. Im Januar, der auch unter die vorgenannten 7 Monate zu rechnen ist, konnte ich solche nicht finden.

Bei 73 Nasen, welche ich in 9 Monaten öffnete, fand ich in 8 Monaten regelmässig Distomum globiporum Rud., im Januar konnte ich niemals das Vorhandensein irgend einer Distomumspecies constatiren.

In 8 Monaten waren 73 Alet Gegenstand meiner Untersuchung. Während der drei aufeinanderfolgenden kälteren Monate Januar, Februar und März fand ich kein einziges Exemplar mit Trematoden inficirt.

Nur beim Flussbarsch, dessen Nahrungsaufnahme sich während der kalten Jahreszeit nur reducirt, nicht aber ganz eingestellt wird, fand ich auch im Januar dieselben Trematoden wie in anderen Monaten.

Dessenungeachtet wird die folgende Behauptung als richtig erachtet werden müssen : Da mit Eintritt der kälteren Jahreszeit die Fische keine oder nur sehr wenig Nahrung zu sich nehmen, so macht sich auch in dieser Zeit ein Mangel an Fischtrematoden bemerkbar, ebenso dürfte bei jenen Fischen, die in Folge der zu grossen Wasserwärme keine Nahrung zu sich nehmen, auch ein Einfluss auf den Bestand der Trematoden ausgeübt werden. 


\section{c) Einfluss der Fortpflanzungsperiode des Wirtes auf seine Schmarotzer.}

Das geringe Auftreten oder Fehlen von Trematoden in Fischen zu bestimmten Zeiten des Jahres hat auch noch andere Gründe. Die Zeit der Fortpflanzung nämlich macht ihren Einfluss auch auf die Schmarotzer geltend.

Ein hiesiger Fischzüchter, Herr Friederich GLASER Sohn, teilte mir in freundlichster Weise mit, dass die Forellen, die er hauptsächlich züchtet, während der Laichzeit weniger Nahrung als sonst zu sich nehmen, dagegen sollen dieselben nach dieser Periode eine ausserordentliche Gefrässigkeit zeigen. Vielleicht lässt sich diesèr Befund verallgemeinern, es wäre damit erklärt, wesshalb die Süsswasserfische während der Laichzeit einen geringeren Trematodenbestand aufweisen als sonst.

So hat z. B. ZschokKe $(1889,1891)$ nachgewiesen, dass die Parasitenzahl von Trutta salar nach Arten und Individuen ihr Minimum während der Laichzeit erreicht. Dies ist aber gerade die Zeit, in welcher der Rheinsalm keine Nahrung zu sich nimmt.

Da ich diese Verhältnisse auch bei meinen Untersuchungen stets berücksichtigt habe, so gebe ich zuerst eine Tabelle, in welcher die Laichzeit der von mir untersuchten Fische nach FAtio's $(1882,1890)$ vorzüglichem Werke graphisch dargestellt ist. 


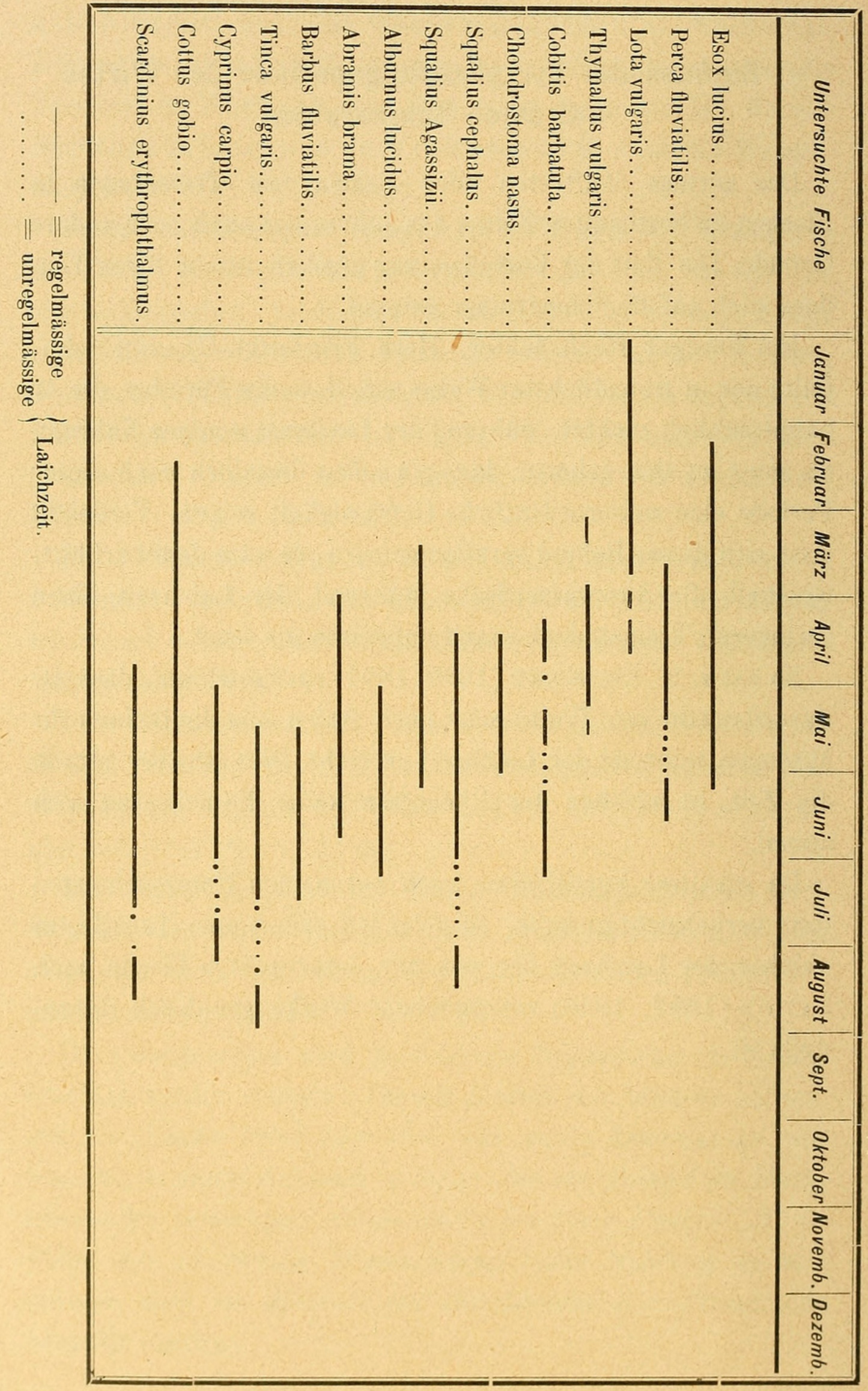


In dieser Liste sind die Wanderfische und diejenigen Süsswasserfische, welche in keinem der Laichmonate untersucht worden sind, weggelassen.

Vergleichen wir diese Zusammenstellung mit dem am Ende des ersten Teiles der Arbeit gegebenen Verzeichniss der Trematodenfunde nach den Monaten eines Jahres, so fällt sofort auf, dass gewisse Fische in den Laichmonaten überhaupt keine Trematoden besitzen, andere dagegen weniger Arten als sonst lieferten.

Esox lucius z. B. beherbergt von Ende Februar bis Ende Mai keine Trematoden. Perca fluviatilis, deren Laichzeit in die Monate April und Mai fällt und sich auch in den Juni erstrecken kann, fand ZschokKe (1884) im April und Juni ohne Parasiten; dagegen fand er im Mai in besagtem Wirt eine Trematodenspecies.

Auch ich habe im Juni in Perca flwiatilis nichts gefunden.

So liesse sich für alle oben angegebenen Fische eine mehr oder weniger starke Reduktion im Auftreten der entoparasitischen Trematoden während der Laichzeit nachweisen; immer aber ist dieselbe begründet in dem verringerten Nahrungsbedürfniss der Fische während der Fortpflanzungsperiode.

\section{d) Einfluss des Wohnortes des Wirtes auf seine Schmarotzer.}

Eine andere äussere Bedingung, welche für die Parasitenfauna eines Fisches nicht ohne merklichen Einfluss ist, haben wir in der Beschaffenheit des Mediums zu suchen, in welchem der Parasitenträger lebt. Dass dasselbe an verschiedenen Orten verschieden sein wird, ist leicht einzusehen. Das Leben im See ist ein anderes als im Fluss, das Wohnen im Teich ein anderes als im Aquarium.

Wie mir Herr GLASER mitgeteilt hat, so nehmen viele Süss- 
wasserfische in der Gefangenschaft keine Nahrung zu sich. Dies fand ich auch bestätigt, nachdem ich den Darmtractus von Fischen, die längere Zeit im Aquarium gelebt hatten, vollständig leer antraf.

Das kann nun nicht ohne Einfluss auf die Parasiten bleiben. In der That weist schon Monticelli (1888) darauf hin, dass die meisten Plagiostomen nach-längerem Aufenthalt im Aquarium ihre Schmarotzer verlieren. Ebenso hat ZschoKKE (1889) die Beobachtung gemacht, dass «sonst parasitenreiche Meerfische der verschiedensten Art, in der Gefangenschaft einer Hungerkur unterworfen, verhältnissmässig schnell ihrer Darmschmarotzer verlustig gehen ».

Es taucht nun die Frage auf, ob die Parasiten selbstständig auswandern oder ob sie mit der Entleerung des Darmkanales nach aussen befördert werden. Es ist beides möglich.

Von einem Auswandern der Trematoden berichtet BRAUN (1890). Er hat beobachtet, dass aus Fröschen, deren Wohnort verändert worden war, Distomum cylindriaceum Zed. auswanderte. Die Tierchen liessen, vermutlich zum Zwecke der Erhaltung der Species, massenhaft Eier frei werden.

Ebenso erwähnt Looss (1894), dass Distomum tereticolle Rud. und nodulosum Zed. ihren Wirt verlassen, jedoch erst wenn derselbe todt ist. Auch ich machte die Beobachtung, wenn auch nicht an Distomen, so doch an Tænien, dass solche aus lebenden Stichlingen im Aquarium auswanderten.

Wir dürfen also annehmen, dass ein Fisch seinem freien Leben entrückt, und in Gefangenschaft gehalten, infolge Einstellens der Nahrungsaufnahme seine Trematoden verlieren kann und zwar oft durch eine Auswanderung derselben.

Für die Verbreitung der Trematoden ist auch die geographische Lage des Wohnortes des Wirtes von Wichtigkeit.

In dieser Beziehung hat zuerst R. LEUcKaRT (1863) Untersuchungen angestellt. Er fand dabei, dass die geographische 
UEBER TREMATODEN DER SÜSSWASSERFISCHE.

Verbreitung der menschlichen Parasiten weniger durch den Menschen, als durch die Zwischenwirte seiner Schmarotzer bedingt ist.

Für Fischparasiten ist Prenant (1886) zu folgendem Schluss gelangt: «La distribution géographique d'un ver parasite a pour facteurs celle du poisson, son hôte, et celle de la pâture de cet hôte.»

Durch meine Untersuchungen glaube ich annehmen zu müssen, dass der geographischen Verbreitung gewisser entoparasitischer Trematodenarten eine Grenze gezogen ist

Manche Arten sind an einem Orte selten oder gar nicht zu treffen, die sich sonst an anderen Stellen regelmässig vorfinden.

Distomum tereticolle Rud. fand JuRINe (1823), der ohne Zweifel Hechte des Genfersees untersucht hatte und ZschokKE (1884), der dasselbe that, in besagten Fischen sehr häufig, dagegen traf Prenant (1886) bei Durchsuchung von 51 Flusshechten dasselbe Distomum nur dreimal an. Er vermutet desshalb, dass Distomum tereticolle Rud. in Hechten, welche grossen Seen entstammen, weit häufiger ist als in Flusshechten.

Dasselbe Distomum findet sich ausserdem noch in anderen Fischen des Genfersees, in der Basler Fischfauna scheint es jedoch nur als Bewohner des Hechtes aufzutreten, wenigstens fand ich es nur in diesem Wirt und zwar in 36 Exemplaren 7 Mal.

Auch von Distomum folium Olfers liesse sich Aehnliches sagen. Während es in den Fischen des Genfersees selten vermisst wird, fand ich es in der Basler Fauna überhaupt nicht.

So bleiben also selbst gesetzmässige Träger eines Distomum's in bestimmten Gegenden von ihrem Schmarotzer ganz verschont und daran ist allein die geographische Verbreitung des Zwischenwirtes des Parasiten schuld.

Zwischenwirte der Fischtrematoden sind meistens Mollusken. Nun ist aber gerade die Molluskenfauna von Ort zu Ort variabel 
und demnach muss auch die Verteilung der Zwischenwirte der Fischtrematoden und damit die Trematodenfauna der Fische überhaupt in den verschiedenen Gegenden eine verschiedene sein.

Der Wohnort eines Fisches, um noch kurz zusammenzufassen, ist nicht ohne Einfluss auf seine Schmarotzer. Ein Fisch, aus dem Freien z. B. in die Gefangenschaft gebracht, verliert in der Regel seine Parasiten infolge Einstellens der Ernährung.

Mit der geographischen Lage des Wohnortes eines Fisches, ändert sich auch seine Parasitenfauna und zwar ist diese Aenderung bedingt durch die geographische Verteilung der Zwischenwirte der Parasiten.

\section{Verschiedene biologische Bemerkungen.}

\section{a) Die gegenseitige Verdrængung der Parasiten.}

Es ist eine bekannte Thatsache, dass ein Fisch verschiedene Schmarotzer zugleich beherbergen kann; dass aber das Eindringen der einen eine Verdrängung der anderen zur Folge haben kann, ist bis jetzt noch wenig beachtet worden.

Prenant (1886) z. B. spricht von einem Antagonismus des Trienophorus und des Echinorhynchus; ich kann Aehnliches von Echinorhynchus und Distomum berichten.

Die 137 von mir untersuchten Barben nämlich lieferten nur neun Mal Distomen, die übrigen waren zumeist mit Echinorhynchen behaftet und zwar in solchem Maasse, dass der Darm von ihnen völlig "gespickt» war. Wo nun ein solch, massenhaftes Auftreten der Echinorhynchen stattfand, waren die Distomen zurückgedrängt und vertrieben. Dass die beiden Formen überhaupt nicht miteinander leben können, soll damit nicht gesagt sein; immerhin fand ich, dass, bei Vorhandensein einer grossen Menge von Echinorhynchen, Distomen zu gleicher 
Zeit nicht zu finden waren, während letztere dann in grosser Zahl auftraten, wenn die Echinorhynchen sich nur spärlich zeigten.

Zum Beweis kann ich noch ein weiteres Beispiel anführen.

Bei 134 Aeschen, die mir zur Beobachtung vorlagen, stellten die Echinorhynchen immer ein grosses Contingent zum Parasitenheer dieser Fische. Nur acht Mal fand ich Distomen, mit denen stets nur wenige Echinorhynchen zusammen waren.

\section{b) Das gesetzmæssige Auftreten der Trematoden.}

Durch eine Vergleichung der Angaben über Trematodenfunde habe ich mich überzeugt, dass sich die Individuenzahl, in welcher eine Trematodenspecies ihren Wirt bevölkert in ganz gesetzmässigen Grenzen hält.

Gewisse Arten treten nur einzeln auf, andere in grösserer Anzahl und wieder andere in Massen. So findet sich z. B. Distomum tereticolle Rud. meist nur in Gruppen von 4 bis 5 Individuen zu gleicher Zeit in demselben Wirt. Das Maximum der Individuenzahl überschreitet 15 nicht. Ich übergehe dabei allerdings JuRINE's (1823) Angaben, wonach er bis 30 Exemplare von Distomum tereticolle Rud. zugleich gefunden haben will.

Auch Distomum isoporum Looss scheint die vorhin angegebene Maximalzahl nicht zu überschreiten, wenigstens fand ich es nur einmal in 11 Exemplaren in demselben Wirt.

Distomum farionis Müller ist ebenfalls bis jetzt nur in geringer Zahl in ein und demselben Wirt gefunden worden. Trotzdem es allgemein als für Salmoniden typisch angenommen wird, förderte ich es aus 134 Aeschen nur zwei Mal in der Zahl von zwei bis drei zu Tage.

Aehnliches lässt sich von Distomum globiporum Rud. sagen. Ich hatte Gelegenheit viele Cypriniden, deren typischer Schma- 
rotzer Distomum globiporum Rud. ist, zu untersuchen, niemals aber fand ich es in grosser Zahl beisammen, was auch mit den Angaben verschiedener Autoren stimmt.

Während die bis jetzt genannten Distomen einzeln oder nur in geringer Anzahl miteinander vorkommen, bevölkern andere Arten wie Distomum perlatum v. Nordm. und Distomum nodulosum Zed. den Darm ihrer Wirte in grossen Massen. Vom ersteren fand ich bis zu 50 Individuen in demselben Wirt; von Distomum nodulosum Zed. dagegen zählte ich im Darm einer einzigen Perca fluviatilis 100 Exemplare. Ob auch hier eine Gesetzmässigkeit des Vorkommens herrscht, vermag ich nicht $\mathrm{zu}$ entscheiden; sicher ist nur, dass sich die Zahl der in einem Wirte zugleich schmarotzenden Individuen gewisser Distomenarten in gesetzmässigen Grenzen hält.

\section{c) Das Vorkommen der Trematoden.}

Was die Menge betrifft, in welcher ich Trematoden in der Basler Fischfauna gefunden habe, so lässt sich nur sagen, dass die Fische des Rheines bei Basel verhältnissmässig arm an Saugwürmern sind. Aus 1029 Fischen erhielt ich bei meinen Untersuchungen nur 18 Trematodenarten.

Piesbergen (1886), der die Fischfauna Tübingen's untersucht hat, kommt für jene Gegend zu demselben Ergebniss.

Die genaueren Verhältnisse werden aus folgender Tabelle ersichtlich sein. 


\begin{tabular}{|c|c|c|c|}
\hline Namen der untersuchten Fische & $\begin{array}{c}\text { Anzahl } \\
\text { der } \\
\text { Exemplare }\end{array}$ & $\begin{array}{l}\text { Anzahl der } \\
\text { inficirten } \\
\text { Exemplare }\end{array}$ & $\begin{array}{c}\text { Procente } \\
\text { der } \\
\text { Infection }\end{array}$ \\
\hline 1. Petromyzon fluviatilis............. & 1 & - & $-\%$ \\
\hline 2. Anguilla vulgaris................ & 4 & - & - \\
\hline 3. Alosa vulgaris................. & 1 & 1 & 100 \\
\hline 4. Esox lucius................. & 36 & 7 & 19.4 \\
\hline ร. Trutta salar....... & 20 & 13 & 63 \\
\hline 6. Trutta lacustris.............. & 1 & - & - \\
\hline 7. Trutta fario................... & $1 \check{2}$ & - & - \\
\hline 8. Salmo salvelinus............... & 6 & - & - \\
\hline 9. Thymallus vulgaris. ............. & 134 & 8 & 6 \\
\hline 10. Cobitis barbatula................ & 33 & 3 & 9 \\
\hline 11. Chondrostoma nasus............. & 73 & 18 & 24.6 \\
\hline 12. Phoxinus lævis................. & 45 & 3 & 6.6 \\
\hline 13. Squalius cephalus............... & 73 & 2 & 2.7 \\
\hline 14. Squalius leuciscus................ & 84 & 4 & 4.8 \\
\hline 15. Squalius Agassizii............... & 20 & - & - \\
\hline 16. Scardinius erythrophthalmus........ & 32 & 1 & 3 \\
\hline 17. Alburnus lucidus................ & 11 & - & - \\
\hline 18. Blicca björkna................. & 11 & - & - \\
\hline 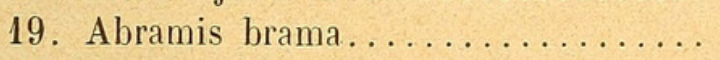 & 26 & 7 & 27 \\
\hline 20. Gobio fluviatilis................ & 19 & 2 & 10.3 \\
\hline 21. Barbus fluviatilis................ & 137 & 9 & 6.6 \\
\hline 22. Tinea vulgaris................ & 39 & 21 & ठั4 \\
\hline 23. Cyprinus carpio............... & 50 & - & - \\
\hline 24. Lota vulgaris.................. & 1 & - & - \\
\hline 25. Cottus gobio.................. & 50 & 4 & 8 \\
\hline 26. Gasterosteus gymnurus............. & 32 & - & - \\
\hline 27. Perca fluviatilis................ & 60 & 8 & 13.3 \\
\hline 28. Acerina cernua ............... & 8 & - & - \\
\hline 29. Lucioperca sandra.............. & 7 & 6 & 86 \\
\hline
\end{tabular}

Von den 1029 Fischen, die ich untersucht habe, waren also 117 Individuen, d. h. 11,4\%, mit Trematoden behaftet.

Da es nicht ohne Interesse ist zu wissen, welche Organe von diesen Parasiten im Wirt bewohnt werden, so will ich hier die nachfolgende Zusammenstellung anfügen. 


\begin{tabular}{|c|c|}
\hline Parasit & Aufenthaltsort \\
\hline 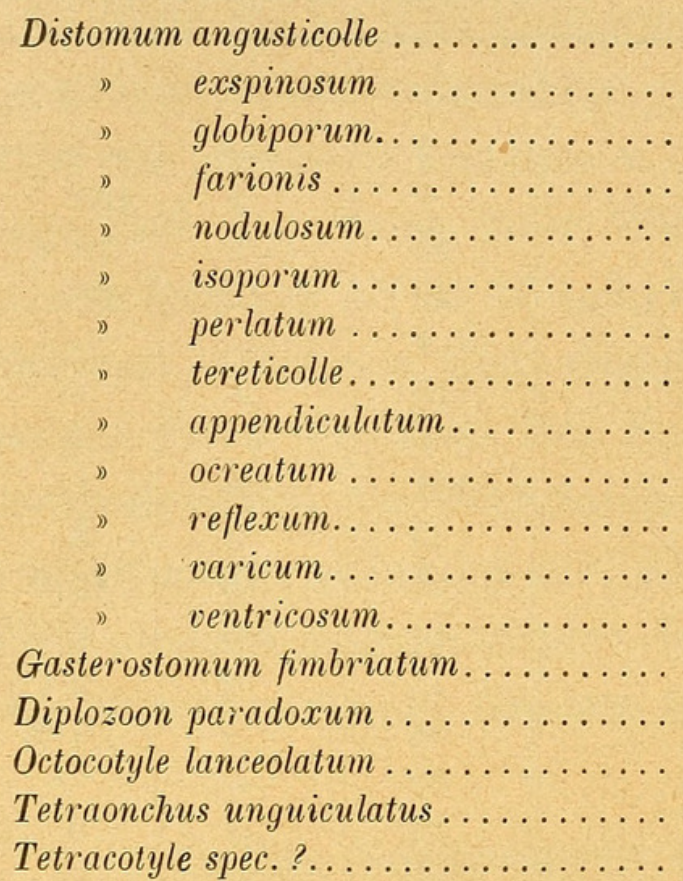 & $\begin{array}{l}\text { Intestinum } \\
\text { Oesophagus, Ventrikel } \\
\text { Intestinum } \\
\text { Ventrikel } \\
\text { Ventrikel, Intestinum } \\
\text { " Intestinum } \\
\text { Branchien } \\
\text { Ad " } \\
\text { Peritoneum in caps. }\end{array}$ \\
\hline
\end{tabular}

Aus Vorstehendem ergibt sich, dass das Intestinum der Hauptaufenthaltsort der entoparasitischen Trematoden der Süsswasserfische ist.

\section{d) Die Lebensdauer der Trematoden ausserhalb ihrer Wirte.}

Durch einige Versuche habe ich der Frage näher zu treten gesucht, wie lange wohl die Distomen ausserhalb ihrer Wirte $z u$ leben im Stande sind und wie weit das Sauerstoffbedürfniss dieser Würmer reiche.

Letzteres war mir von besonderem Interesse, da bereits BungE $(1883,1890)$ erfolgreiche und wichtige Versuche über den Sauerstoffbedarf anderer Darmparasiten vorgenommen hat.

Die Zahl meiner Versuche ist allerdings eine geringe, dennoch teile ich meine Resultate in Kürze mit. 
Als Versuchsobjekt diente mir Distomum globiporum Rud. Von acht Exemplaren lebten die vier, welchen der Sauerstoff vollständig entzogen worden war, drei bis vier Mal 24 Stunden, während die anderen vier, denen es an Sauerstoff nicht fehlte, nur zwei Mal 24 Stunden weiter lebten. Diese letzteren waren in gewöhnliches Brunnenwasser gelegt worden.

Frische Exemplare desselben Distomum blieben, in $1 \%$ Kochsalzlösung gebracht, nach Sauerstoffentzug fünf Mal 24 Stunden am Leben, ohne Entfernung des Sauerstoffes jedoch nur drei bis vier Mal 24 Stunden.

Ausserdem behielten sämtliche Versuchsobjekte in sauerstofffreien Medien nicht nur ihre Lebhaftigkeit, sondern auch ihre Farbe länger bei als die andern.

Andere Distomen scheinen noch lebenszäher zu sein als Distomum globiporum Rud.; so erhielt J. V.AN BENEDEN (1858) acht Tage lang Distomum tereticolle Rud. in Wasser lebend.

JURINE (1823) will sogar dasselbe Tier einen Monat lang ausserhalb des Wirtes lebend befunden haben.

Die Richtigkeit dieser Angabe könnten nur weitere Versuche beweisen, dagegen stimmen meine Resultate mit denen von BUNGE $(1883,1890)$ überein, wonach sich bei Entoparasiten der Sauerstoffbedarf auf ein Minimum reducirt. 


\section{Tabelle der Ergebnisse an Trematoden}

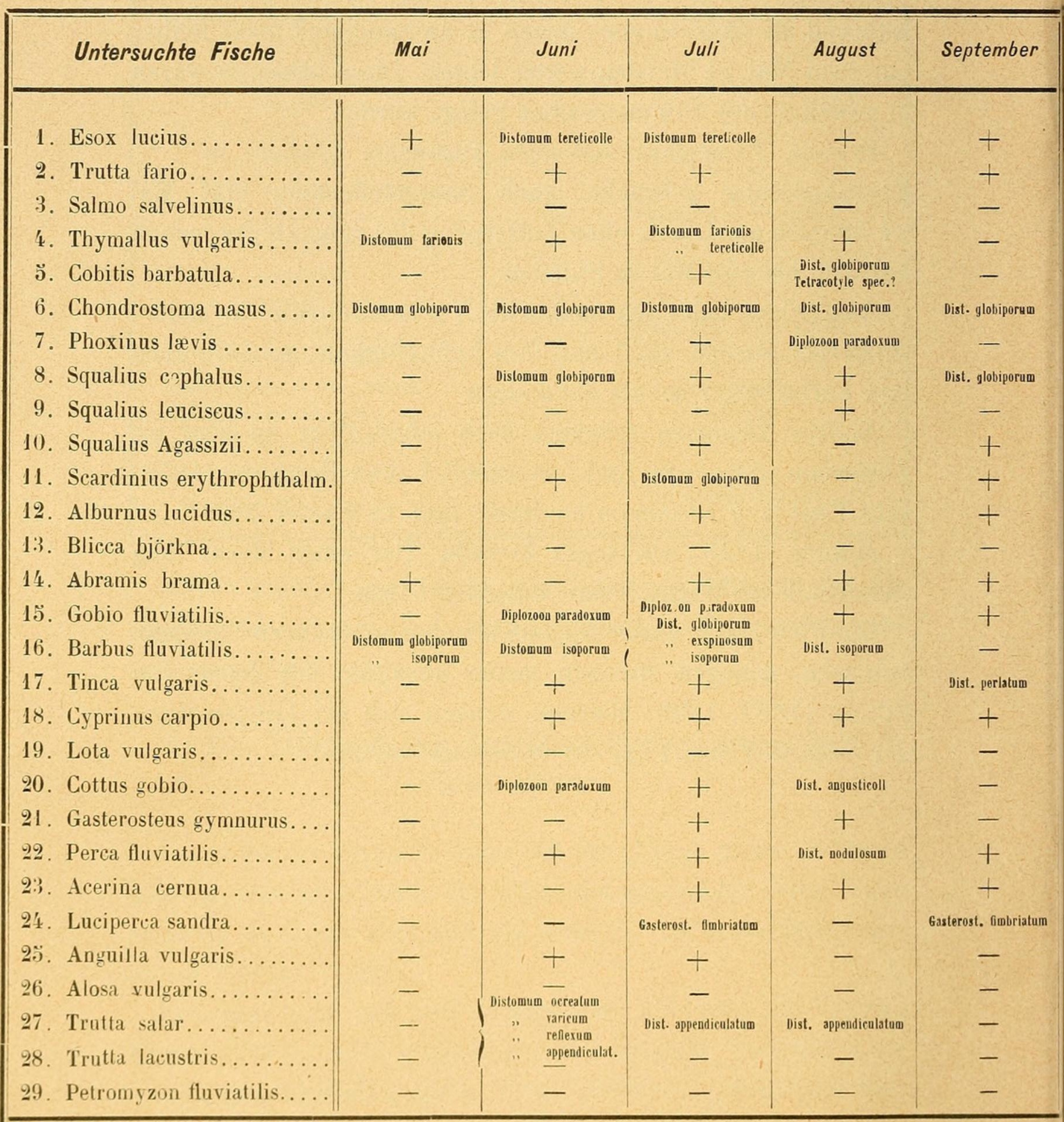


in den einzelnen Monaten.

\begin{tabular}{|c|c|c|c|c|c|c|}
\hline Oktober & November & Dezember & Januar & Februar & März & April \\
\hline Dist. terelico.le & + & - & + & - & + & - \\
\hline- & - & - & + & - & - & - \\
\hline+ & - & - & + & - & - & - \\
\hline+ & + & - & + & - & - & - \\
\hline- & - & - & - & - & - & - \\
\hline- & Dist. globiporum & - & + & - & Disl globiporum & Disl. globiporum \\
\hline- & - & - & - & - & + & - \\
\hline- & + & - & + & + & + & - \\
\hline- & Dist. globiporum & - & - & + & - & - \\
\hline- & + & - & + & + & + & - \\
\hline- & - & - & - & - & - & - \\
\hline- & + & - & - & - & - & - \\
\hline- & + & - & - & - & + & - \\
\hline - & + & Diplozoon paradoxum & - & Dipl. paradorum & $\begin{array}{l}\text { Dist. isoporum } \\
\text { Dipl. paradousum }\end{array}$ & - \\
\hline- & + & + & - & - & - & - \\
\hline - & $\begin{array}{l}\text { Dist. exspinosum } \\
\text {-. perlatum }\end{array}$ & - & + & - & Dist. globiporum & - \\
\hline- & - & Dist. perla'um & + & Dist. perlatum & - & - \\
\hline+ & + & + & + & + & - & - \\
\hline- & - & - & - & - & + & - \\
\hline - & - & - & - & - & + & - \\
\hline- & - & - & - & - & - & - \\
\hline Dist. nodulosun & + & - & Dist. nodulosum & + & -- & - \\
\hline+ & + & - & - & - & - & - \\
\hline - & - & Gast. fimbratam & - & - & Tetraonchus unguiculatus & $\begin{array}{l}\text { Gast. fimbriatum } \\
\text { Tetraonclius unguiculalus }\end{array}$ \\
\hline- & - & - & - & - & - & - \\
\hline- & $\begin{array}{l}\text { Dist. réntricosum } \\
\text { Octocot!le lanceolatum }\end{array}$ & - & - & - & - & - \\
\hline- & - & - & - & + & - & - \\
\hline- & - & - & - & + & - & - \\
\hline- & - & - & - & - & - & + \\
\hline
\end{tabular}


II.

\section{Speziellep Tril.}

\section{Distomum angusticolle nov. spec.}

Fig. 1-3.

Allgemeines und æussere Kœrperform. Dieses Distomum habe ich (1896) im Intestinum zweier Cottus gobio L. am 30. August 1895 gefunden. Die übrigen der von mir untersuchten 39 Groppen beherbergten diesen Schmarotzer nicht.

Distomum angusticolle ist dem Subgenus Dicrocœlium Duj. zuzuteilen, d. h. denjenigen unbewaffneten Distomen, welche einen Darm mit einfachen, verlärgerten Schenkeln besitzen, deren Bauchsaugnapf den Mundsaugnapf an Grösse übertrifft und ohne gestielt zu sein doch etwas über die Bauchfläche hervorragt. In diesem Subgenus ist es denjenigen Formen anzureihen, deren Hoden hinter den Uterusschlingen gelegen sind. Da die neue Art von ihren nächsten Verwandten in verschiedenen Punkten wesentlich abweicht, so werde ich eine kurze Beschreibung folgen lassen. Ich schlage vor die neue Species Distomum angusticolle zu nennen.

Was die Gestalt dieses Wurmes anbetrifft, so zeichnet er sich besonders dadurch aus, dass dem kurzen, etwas gebogenen schmalen Halsteil ein bedeutend breiterer, in gerader Richtung verlaufender Körper folgt, welcher am Hinterende ovalartig abgerundet ist. Dieses ist auch in den Naassen ausgedrückt.

Die Länge des ganzen Tieres beträgt 1,5 mm., die Breite um den Pharynx 0,13 mm. und bei den Hoden 0,30 mm.

Ein auffallender Unterschied ist in der Grösse der beiden Saugnäpfe gegeben. Der Durchmesser des Mundsaugnapfes 
beträgt 0,09 mm., derjenige des Bauchsaugnapfes dagegen $0,22 \mathrm{~mm}$.

Die Farbe des Tieres ist weisslich-grau.

Kœrperdecke. Die Haut von Distomum angusticolle ist glatt und liegt dem ganzen Körper fast gleichmässig an. Es lassen sich an ihr zwei Schichten deutlich unterscheiden. Die äussere ist hyalin, während die innere eine feinkörnige Masse darstellt.

Saugnæpfe. Die Maasse dieser Haftapparate sind bereits oben angegeben worden, es ist aus ihnen ersichtiich, dass der Bauchsaugnapf doppelt so gross ist als der Kopfsaugnapf.

Auf die Morphologie und Histologie der Saugnäpfe gehe ich nicht näher ein, da hierüber schon ausführliche Beschreibungen existiren und Distomum angusticolle in dieser Hinsicht nicht vom Allgemeinen abweicht.

Ich möchte nur noch auf die Einlagerung grosser Zellen im Kopfsaugnapf aufmerksam machen. Es wurden dieselben schon von andern Arten beschrieben, jedoch wissen wir bis heute nicht, welche Bedeutung dieselben haben.

Verdauungssystem. Auf den Mundsaugnapf folgt, durch einen kurzen Vorhof getrennt, der $0,06 \mathrm{~mm}$. breite muskulöse Pharynx. Derselbe ist wohl ausgebildet und zeigt aussen eine Längsmuskulatur, an die sich eine Schicht von Ringmuskeln reiht. Nach innen wird der Pharynx von einer cuticulaartigen Membran begrenzt. Auch im Pharynx sind einige jener grossen Zellen fraglicher Natur eingebettet. Nach unten zu ist er abgerundet.

Der Oesophagus ist $0,016 \mathrm{~mm}$. breit und $0,05 \mathrm{~mm}$. lang, derselbe lässt seinen Zusammenhang mit dem Pharynx und dem Vorhof deutlich erkennen. Besonders stark ist die Ringmuskulatur desselben ausgebildet, jedenfalls zu dem Zwecke die aufgenommene Nahrung in die beiden Darmschenkel zu treiben. An der Ursprungsstelle dieser beiden letzteren ist der Oesophagus ziemlich breit. Die Gabelung beginnt etwas vor dem Bauch- 
saugnapf. Die beiden Darmäste erstrecken sich bis in die Nähe des Körperendes, sie reichen nur ein kleines Stück weit über den hinteren Hoden hinaus und endigen blind.

In histologischer Beziehung ist der Darm, der von allen Seiten von Dotterfollikeln umgeben ist, folgendermassen zusammengesetzt (Fig. 2). Zuäusserst zieht sich eine Längsmuskellage, daran reiht sich eine Ringfaserschicht und nach innen folgt das Epithel. Die Ringmuskelfasern stehen fast so dicht beisammen wie im Oesophagus.

Nervensystem. Dasselbe ist besonders in seiner vorderen Partie deutlich zu erkennen. Zwischen Mundsaugnapf und Pharynx liegen die beiden Gehirnganglien, die durch eine kräftige Commissur miteinander verbunden sind. Letztere ist sehr breit und misst $0,01 \mathrm{~mm}$. Das Nervensystem ist scharf gegen das umgebende Parenchym abgegrenzt. Da der Körper fast ganz von den Dotterstöcken erfüllt wird, so war die Untersuchung der übrigen Teile des Nervensystems mir nicht möglich.

Excretionssystem. Aus dem soeben angegebenen Grunde war auch das Exkretionssystem nur teilweise zu beobachten. Zum Porus excretorius führt ein länglicher Kanal, der wohl als Sammelkanal bezeichnet werden kann. Die Oeffnung des Exkretionsporus misst im Durchmesser 0,02 mm.

Genitalsystem. Die Geschlechtsöffnungen sind vor dem Bauchsaugnapf gelegen und zwar aus der Mittellinie mehr nach rechts gerückt in die Nähe der Gabelungsstelle des Darmes.

Männlicher Apparat. - Die Hoden des Distomum angusticolle bilden zwei ganzrandige, ansehnliche Körper, die dicht hintereinander gelagert sind. Sie nehmen fast die ganze Breite des Tieres ein und liegen in der hinteren Körperhälfte. Sie erstrecken sich über das dritte Viertel des Körpers und noch etwas darüber hinaus. Die Länge des einzelnen Organes beträgt $0,22 \mathrm{~mm}$., die Breite $0,18 \mathrm{~mm}$. Die Hoden sind von einer dünnen Membran umgeben. Im Innern zeigen sich kleinere, 
peripherische und grössere, centrale Zellen, woraus zu schliessen wäre, dass dieselben von aussen nach innen sich ablösen. Die Spermatozoen sind oft in Büscheln angeordnet.

Aus den beiden Hoden kommen von dem oberen Teil derselben als Fortsetzung der Membrana propria die beiden Vasa efferentia. Beim Eintritt in den Cirrusbeutel vereinigen sich dieselben zum Vas deferens. Letzteres bildet eine grosse Vesicula seminalis, welche stark mit Sperma angefüllt wird. Die Samenblase ist vom Cirrusbeutel vollständig umschlossen. Im Cirrus findet der männliche Leitungsapparat sein Ende.

Weiblicher Apparat. - Der Keimstock ist links vor dem vorderen Hoden gelegen. Auch er hat wie die Hoden kugelförmige Gestalt. Er ist $0,16 \mathrm{~mm}$. lang und $0,13 \mathrm{~mm}$. breit; eingeschlossen wird er von einer stark glänzenden, homogenen Membran. Am Rande zeigen sich kleinere, nach innen zu immer grössere Keimzellen. An seiner Ausmündungsstelle ist der Keimstock etwas buckelförmig ausgesackt. In dieser kleinen Erhebung liegen ganz reife Keimzellen, jedenfalls solche die zunächst zur Verwendung bereit sind. Aehnliche Verhältnisse finden wir auch noch bei anderen Distomen.

Direkt nach seinem Austritt empfängt der Keimgang den LAURER'schen Kanal mit dem angehängten Receptaculum seminis. Der LAURER'sche Kanal ist sehr deutlich zu erkennen, er biegt ein wenig nach hinten aus, verläuft dann nach oben und mündet auf dem Rücken aus. Das Receptaculum seminis ist gut entwickelt und wird reichlich mit Sperma angefüllt, während der Kanal leer angetroffen wurde. Die Länge des Receptaculums beträgt $0,14 \mathrm{~mm}$. und die Breite $0,072 \mathrm{~mm}$. In seiner Lage fällt es nur teilweise mit dem Keimstock zusammen. Wenn es strotzend mit Sperma erfüllt ist, so stellt es sich als ein undurchsichtiger Körper dar. Distomum angusticolle wäre zu jenen Formen zu rechnen, die ein besonders grosses Receptaculum seminis besitzen, also zu Distomum nodulosum Zed., isoporum Looss und variegatum Rud. 
Nach erfolgter Aufnahme des LaURER'schen Kanals tritt an den Keimgang der Dotterkanal heran, der aus einem kleinen Dotterreservoir hervorkommt.

Die Dotterstöcke, welche eines der Haupterkennungszeichen dieser Species bilden, sind enorm entwickelt und zwar in einem Maasse, wie sie bei wenigen Formen der Süsswasserdistomen bis jetzt gefunden worden sind.

In der Regel sind die Dotterstöcke seitlich angeordnet, bei unserer Form dagegen sind sie fast über den ganzen Körper ausgebreitet, sodass jeder Raum, der nicht von anderen Organen beansprucht wird, von Dotterfollikeln angefüllt ist.

Die Eier von Distomum angusticolle (Fig. 3) sind 0,055$0,06 \mathrm{~mm}$. gross. In dieser Hinsicht dürfte unsere Species in dem von DuJaRdiN (1845) aufgestellten Verzeichniss der Distomen nach Eigrösse zwischen Distomum flexuosum Rud. und Distomum filum Duj. einzureihen sein.

Trotzdem sich bei Untersuchung des geschlechtsreifen Tieres wenig Eier vorfanden, so muss doch die enorme Entwicklung der Dotterstöcke auch eine reichliche Eiproduktion annehmen lassen. Wahrscheinlich werden eben die Eier ziemlich frühe abgegeben, denn ich fand stets nur Eier in noch wenig fortgeschrittener Embryonalentwicklung. Die Eier sind oval.

Dieselben gelangen vom Keimstock in den Keimgang, welcher sie durch ein Ootyp dem kleinen Uterus zuführt. Dieser legt sich fast rings um den Bauchsaugnapf herum und steigt unter demselben hinan, um neben der männlichen Geschlechtsöffnung zu münden.

Wie wir schon gesehen haben, sind für Distomum angusticolle die Dotterstöcke in ihrer Ausbildung charakteristisch. Sie bleiben nicht nur zu beiden Seiten, sondern verschmelzen gleichsam im Vorder- und Hinterende des Körpers. Auch von anderen Distomen ist diese Ausbildung der Dotterstöcke bekannt, so besonders von Distomum mormyris Stoss. (1885). Doch ist es 
nicht möglich, die neue Species mit der letzteren zu identificieren, ebensowenig mit andern Distomenarten, die sich im oben genannten Characteristicum dem Distomum angusticolle nähern.

Fassen wir das Wichtigste aus vorliegender Beschreibung zusammen, so lässt sich über Distomum angusticolle folgendes sagen.

Das zur Gruppe Dicroccelium gehörige Distomum ist unbestachelt, besitzt einen kleinen Mundsaugnapf, einen doppelt so grossen Bauchsaugnapf. Der Pharynx und Darm ist muskulüs, letzterer teilt sich vor dem Bauchsaugnapf in die beiden Schenkel, die sich fast bis ans Körperende erstrecken. Die beiden Gehirnganglien sind scharf ausgeprägt und durch eine kräftige Kommissur miteinander verbunden. Der Exkretionsapparat bildet vor seinem Austritt einen länglichen Sammelkanal. Die Geschlechtsöffnungen sind vor dem Bauchsaugnapf gelegen und zwar aus der Mittellinie nach rechts verschoben. Die beiden kugeligen grossen Hoden liegen im dritten Viertel des Körpers. Der Keimstock liegt vor den Hoden, sein Ausführgang bildet ein Ootyp und empfängt die beiden Dottergänge, welche von den äusserst stark entwickelten Dotterstöcken kommen. Die Eier gelangen nach der Befruchtung in einen kleinen, wenig gewundenen Uterus, welcher neben der männlichen Geschlechtsöffnung ausmündet.

2. Distomum perlatum v. Nordm. var. exspinosum var. nov.

Fig. 4-วั.

Allgemeines und æussere Kœrperform. Das vorliegende Distomum ist von mir desshalb als neue Varietät der Species Distomum perlatum v. Nordm. aufgestellt worden, weil dasselbe grosse Aehnlichkeit mit Letzterem zeigt, jedoch in einigen, nicht unwesentlichen Punkten von der Stammform abweicht. 
Da die Varietät im Gegensatz zur Art unbestachelt ist, so schlage ich vor, dieselbe exspinosum zu nennen.

Manche Uebereinstimmung zeigt Distomum punctum Zeder (1803) mit unserer Varietät, jedoch sind die Angaben über die letztgenannte Art so allgemeiner Natur, dass eine Identificirung nicht möglich ist.

Distomum exspinosum habe ich in 137 Barben zwei Mal im Intestinum gefunden. Das eine Mal sammelte ich es am 19. Juli 1895 in 3 Exemplaren, das andere Mal am 23. November desselben Jahres in 2 Individuen. Die Varietät scheint also in der Basler Fauna selten zu sein. Die Barben waren dem Rhein bei Mumpf entnommen worden.

Distomum exspinosum kommt im gleichen Wirt vor wie die von v. Linstow (1877) als Distomum ferruginosum beschriebene und von Looss (1894) mit Distomum perlatum v. Nordm. identificierte Species. Da Distomum ferruginosum v. Linst. auch mit unserer neuen Varietät nichts zu thun hat, so mag die Behauptung von Looss (1894), es sei dasselbe identisch mit Distomum perlatum v. Nordm., wohl richtig sein.

Da das letztere Distomum vom soeben genannten Autor genau beschrieben worden ist, so werde ich von Distomum exspinosum nur die abweichenden Verhältnisse näher besprechen.

Distomum exspinosum wird im Durchschnitt 1,24 mm. lang, seine Farbe ist eine hellgelbe. Distomum perlatum v. Nordm. ist dagegen gelblichrot, Looss (1894) hat sogar in Abramis brama Exemplare gefunden, welche unter der Haut ein « braunrotes Pigment in Gestalt von mehr oder minder grossen und intensiv gefärbten Flecken » bilden.

Kœrperdecke. Die Haut unserer Varietät ist unbestachelt und vollständig glatt. Nicht so bei der Stammform; Distomum perlatum ist vollständig bestachelt und selbst die Endteile des männlichen und weiblichen Leitungsapparates der Geschlechtsprodukte sind mit Stacheln bewehrt. 
UEBER TREMATODEN DER SÜSSWASSERFISCHE.

Selbst wenn man annehmen muss, dass die Stacheln sehr leicht abfallen, so kann dies doch bei Distomum exspinosum nicht der Fall sein, da selbst von einem Stachelbesatz der Geschlechtsöffnung und der daran anschliessenden Kanäle keine Spur zu entdecken ist.

Saugnæpfe. Das Grössenverhältniss der beiden Saugnäpfe ist für Distomum perlatum v. Nordm. ein ganz anderes als für seine Varietät. Bei ersterem hat der Mundsaugnapf einen Durchmesser von 0,15 mm., der Bauchsaugnapf einen solchen von $0,18 \mathrm{~mm}$., bei der letzteren misst der Mundsaugnapf $0,19 \mathrm{~mm}$., der Bauchsaugnapf $0,26 \mathrm{~mm}$.

Da Schwankungen in der Grösse der Saugnäpfe der Distomen nicht selten sind, so will ich bemerken, dass die für Distomum exspinosum angegebenen Zahlen Durchschnittszahlen sind.

Verdauungssystem. Der Digestionsapparat zeigt im Allgemeinen keine erwähnenswerten Besonderheiten. Der Mundsaugnapf führt durch einen kleinen aber ziemlich breiten Vorhof in den Pharynx. Dieser ist sehr gut entwickelt und weist eine stark ausgeprägte Muskulatur auf. Er erreicht fast die Hälfte des Durchmessers des Mundsaugnapfes, während der Pharynx des Distomum perlatum v. Nordm. kaum ein Drittel der Grösse seines Mundsaugnapfes erreicht. Die grossen Zellen, welche ich schon bei Distomum angusticolle erwähnte, treten auch hier im Pharynx deutlich auf.

Der Oesophagus teilt sich kurz vor dem Bauchsaugnapf in die beiden Darmschenkel, welche nur wenig über die Hoden hinaus ragen.

Nerven sy ste m. Das Nervensystem scheint dem allgemeinen Trematodentypus, wie er von GAFFRON (1884) angegeben wird, $\mathrm{zu}$ entsprechen; da ich jedoch dasselbe nicht vollständig beobachten konnte, so will ich nicht weiter darauf eingehen.

Exkretionssystem. Bei keinem der anderen Süsswassertrematoden tritt das Exkretionssystem so auffallend hervor wie 
bei Distomum exspinosum. Zwar ist die Exkretionsblase durch den weit ausgedehnten Hoden, sowie durch die vielen Uterusschlingen verdeckt, vielleicht sieht man sie aber an jüngeren Exemplaren.

Auf beiden Seiten des Körpers verlaufen je zwei Hauptstämme des Exkretionssystems, von denen die ventralen bedeutend weiter sind als die dorsalen. Die ersteren geben, wenn sie in ihrem Verlauf etwa die Mitte der Hoden erreicht haben, je ein Seitengefäss ab. Zwei weitere Seitengefässe entspringen den Hauptstämmen etwa in der Höhe des Bauchsaugnapfes. Die Exkretionsgefässe sind oft von stark lichtbrechenden Körnchen erfüllt.

Genitalsystem. Viel Uebereinstimmendes finden wir im Bau der Genitalapparate bei der Varietät und der Species.

Bei beiden Formen sind die Geschlechtsöffnungen auf den äussersten Körperrand gerückt. Obgleich sie neben dem ventralen Saugnapf liegen, so befindet sich doch die Vesicula seminalis noch hinter demselben.

Der männliche und weibliche Geschlechtsapparat mündet durch einen gemeinschaftlichen Porus nach aussen.

Männlicher Apparat. - Wie Distomum perlatum v. Nordm., so besitzt auch die neue Varietät nur einen Hoden; während derselbe bei ersterem aber ein eiförmiges Gebilde ist, dessen Länge $0,15 \mathrm{~mm}$. und dessen Breite $0,08 \mathrm{~mm}$. beträgt, so hat der Hoden von Distomum exspinosum Kugelform, eine Länge von $0,25 \mathrm{~mm}$. und eine Breite von 0,27 mm. Aus dem Hoden entspringen zwei Vasa efferentia, die sich nach kurzem Verlaufe zur Bildung einer grossen Vesicula seminalis vereinigen. Die Samenblase und der darauffolgende Endteil des männlichen Samenganges werden von einem Cirrusbeutel umschlossen.

Der Ductus ejaculatorius ist nicht wie bei Distomum perlatum v. Nordm. bestachelt, sondern entbehrt jeder Bewaffnung. Wenn er ausgestülpt ist, so nimmt er fast kugelige Gestalt an. 
Sperma findet sich namentlich in der Samenblase reichlich.

Weiblicher Apparat. - Der Keimstock liegt links vor dem Hoden.

Er ist nahezu kugelförmig, nicht etwa gestreckt und oval wie bei der Species. Den LauRER'schen Kanal nebst seinem Receptaculum konnte ich nicht beobachten.

Bei Varietät und Species sind die Dotterstöcke doldenförmige Drüsen, von denen quere Gänge abgehen. Diese Dotterkanäle vereinigen sich in der Mitte des Körpers vor dem Hoden zu einem dreieckigen Dotterreservoir.

Der Uterus nimmt den hinter dem Bauchsaugnapf gelegenen Körperabschnitt ein und endet in der blasig erweiterten, mit eigentümlichen Zellgebilden ausgerüsteten Vagina. Dieselbe ist im Gegensatz zu Distomum perlatum v. Nordm. unbestachelt.

Auch in Bezug auf die Eier zeigen sich zwischen Art und Varietät Unterschiede. Wenn auch die Form der Eier bei Distomum perlatum v. Nordm. schwankend ist, so beträgt das Durchschnittsmaass derselben doch $0,023 \mathrm{~mm}$. in der Länge und 0,012-0,014 mm. in der Breite. ${ }^{7}$ Die Eier von Distomum exspinosum aber sind $0,018 \mathrm{~mm}$. lang und $0,014 \mathrm{~mm}$. breit. Ausserdem tragen die Eier der Species am Hinterende ein mehr oder weniger hervorragendes Knötchen; die Eier von Distomum exspinosum besitzen ein solches Gebilde nicht. Beiden Eiformen dagegen ist das scharf abgesetzte Deckelchen eigen, wie es in Fig. 5 dargestellt worden ist.

Wie wir gesehen haben sind die Verschiedenheiten, welche Distomum perlatum v. Nordm. von seiner Varietät exspinosum trennen, ziemlich tief greifende. - Letztere ist unbestachelt, selbst die Endteile der Geschlechtswege sind unbewehrt. Für beide Formen verschieden ist das Grössenverhältniss der Saugnäpfe, die Grösse des Pharynx in Beziehung auf den Mundsaugnapf, die Gestalt des Hodens und des Ovariums und ausserdem noch die Form und Grösse der Eier. 
Trotzdem habe ich doch keine neue Species schaffen wollen, um nicht einer Vereinfachung der Classification, wie sie für Distomen erwünscht wäre, entgegenzuarbeiten.

3. Gasterostomum fimbriatum v. Siebold. Fig. 6-8.

Allgemeines und æussere Kœrperform. Dieser durch seinen Tentakelapparat ausgezeichnete Süsswassertrematode wurde in sieben von mir untersuchten Zandern (Lucioperca sandra Cuv.) sechs Mal gefunden. Fr erregte durch die auffallende Beschaffenheit seines vorderen Saugnapfes mein besonderes Interesse.

Da Gasterostomum fimbriatum von ZIEGLER (1883) bereits eingehend beschrieben worden ist, so möchte ich nur einige, auf gewisse Ansichten ZIEGLER's (1883) bezugnehmende Beobachtungen hier anführen.

WAGENER (1852) hat diesen Trematoden zuerst genauer beschrieben und abgebildet (1857). Diesing (1858) hat dann später zwei weitere Species Gasterostomum gracilescens Wag. und minimum Stoss. mit Gasterostomum fimbriatum v. Siebold in ein neues Genus Rhipidicotyle vereinigt.

ZiEGLER (1883) erklärt sich die Anhänge am vorderen Saugnapf dadurch, dass « die zwischen den Zügen der Radiärfasern liegenden Parenchymstreifen hervorgepresst werden und die Muskelhaut und die Körperhaut ausstülpen ».

Die mir vorliegenden Exemplare zeigen fast sämtlich, was auch aus der Zeichnung (Fig. 8) und der microphotographischen Aufnahme (Fig. 6) ersichtlich ist, dass der Kopfsaugnapf mit fünf oder sechs grossen Tentakeln ausgestattet ist. An ihrer Basis stehen ebenso viele, ähnlich gebaute nur kleinere Fühler. Es muss desshalb ZIEGLER's (1883) Vermutung unrichtig sein. Für jeden der grossen Kopfanhänge ist eine deutlich sicht- 
bare Oeffnung vorhanden, welche bei der Ausstülpung vom Tentakel ausgefüllt wird. Besonders gut entwickelt ist in den Kopffuhlern die Längsmuskulatur; die Radiärfasern verlaufen in sechs Radien. Die Anordnung der gesamten Tentakelmuskulatur entspricht genau dem, was ZIEGLER (1883) darüber bekannt gemacht hat.

An den Enden der Tentakel befinden sich kleine Saugnäpfe.

Die hohe Differenzierung des Kopfsaugnapfes steht in keinem Verhältniss zu der Ausbildung des Bauchsaugnapfes, der nur durch eine kleine Mundöffnung repräsentiert wird.

Bei Gasterostomum fimbriatum v. Siebold führt der Pharynx an der ventralen Seite in der Mitte des Körpers direkt nach aussen ohne einen Saugnapf zu bilden. Infolge dessen bleibt allein die festsaugende und locomotorische Funktion dem Kopfsaugnapf überlassen. Dass dabei die grossen Tentakel zur Verwendung kommen werden, ist leicht zu begreifen. Was aber die kleinen Fühler für eine Bedeutung haben, konnte ich mir nicht erklären. Vielleicht dienen sie zum Ersatz der grossen Tentakel.

Tentakelartige Bildungen sind bei Trematoden nicht selten. Bei den Monogenea finden sich solche am Vorderende des Körpers, auch bei ihnen ist der Mangel eines Saugnapfes zu constatieren. Bei den Digenea kommen nach BRAUN (1893) nur in Ausnahmefällen tentakelartige Bildungen vor, so bei Distomum nodulosum Zed. und farionis Müller in Form papillenartiger Fortsätze, ferner bei Distomum furcatum Brems., wo sechs fingerartige Fortsätze die Oeffnung des Bauchsaugnapfes umstellen. Es wären noch weitere Fälle zu erwähnen, die meisten derselben werden wohl dadurch zu erklären sein, dass eine erhöhte Saugfähigkeit gefordert wird, oder dass einer der Saugnäpfe infolge Reduktion des andern vermehrte Arbeit zu verrichten hat. 


\section{Tetracotyle spec.?}

Fig. 9-10.

Bei der Untersuchung von 20 Cobitis barbatula L. aus dem Rhein bei Istein fand ich im August massenhaft Cysten im Peritoneum.

Ich versuchte dieselben zu öffnen, allein es gelang mir nicht ohne die Tiere zu verletzen, welche darin eingeschlossen waren. Erst als ich die Objekte mit verdünntem Picrocarmin, das ich 5 Tage lang einwirken liess, gefärbt hatte, gelang es mir brauchbare Präparate anzufertigen.

Den Inhalt der Cysten erkannte ich als Holostomumlarven, die in ihrer Entwicklung schon sehr weit fortgeschritten waren. Ihre Länge betrug durchschnittlich $0,56 \mathrm{~mm}$., ihre Breite 0,3 mm. Deutlich an ihnen zu erkennen ist der Bauchsaugnapf.

Von den Geschlechtsorganen sind bereits die Hoden und das Ovarium in ihrer Anlage vorhanden. Dies lässt auch die beigegebene microphotographische Aufnahme (Fig. 9) deutlich erkennen.

Die Cyste ist ein 0,03 mm. dickes, hartschaliges Gebilde von $0,65 \mathrm{~mm}$. Länge und $0,54 \mathrm{~mm}$. Breite.

Das Vorkommen einer Holostomumlarve in Cobitis barbatula wird schon von v. Linstow (1890) gemeldet. Allein die Maasse und die sonstigen spärlichen Angaben, welche er darüber macht, stimmen mit meiner Cyste nicht überein, ich habe desshalb die Larve in das von BRANDES (1891) aufgestellte Genus Tetracotyle eingereiht.

\section{Diplozoon paradoxum v. Nordm.}

Dieser höchst interessante Parasit ist besonders durch seine auffallende Form schon häufig Gegenstand eingehender Betrach- 
tung geworden. Besonders hat E. ZELLER $(1872,1888)$ wertvolle embryologische und anatomische Studien an diesem Tiere gemacht.

Diplozoon paradoxum v. Nordm. ist ein typischer Ectoparasit der Cypriniden. Ich fand ihn z. B. in den Kiemenblättern von Abramis brama, Cottus gobio, Gobio fluviatilis und Phoxinus lavis und zwar in den verschiedensten Jahreszeiten.

C. Vogt (1841), welcher Diplozoon paradoxum v. Nordm. aus Abramis blicca, Leuciscus prasinus und Gobio fluviatilis untersuchte, bemerkt folgendes : "Es fiel uns gleich auf, dass wir ohne Zweifel drei verschiedene Species dieses merkwürdigen Genus vor uns hatten. » Entgegen diesen Befunden erwähnen die übrigen Autoren immer nur eine Art von Diplozoon. Ich habe desshalb meine Exemplare genau durchgesehen und gefunden, dass nicht alle gleiche Ausbildung erlangt haben. Wie z. B. die Grössenverhältnisse beschaffen sind, werden nachfolgende Zahlen zeigen :

\begin{tabular}{|c|c|c|c|c|c|c|c|c|}
\hline \multirow{6}{*}{$\begin{array}{l}\text { Länge des Vorderkörpers..... } \\
\text { Länge des Hinterkörpers...... } \\
\text { Breite des Vorderkörpers..... } \\
\text { Breite des Hinterkörpers..... }\end{array}$} & \multicolumn{8}{|c|}{ Grossenangaben der Diplozoen aus } \\
\hline & \multicolumn{2}{|c|}{$\begin{array}{c}\text { Abramis } \\
\text { brama }\end{array}$} & \multicolumn{2}{|c|}{ Cottus gobio } & \multicolumn{2}{|c|}{$\begin{array}{c}\text { Govio } \\
\text { fluviatilis }\end{array}$} & \multicolumn{2}{|c|}{$\begin{array}{l}\text { Phoxinus } \\
\text { lævis }\end{array}$} \\
\hline & 2,88 & $\mathrm{~mm}$ & 1,98 & $\mathrm{~mm}$. & 1,29 & $\mathrm{~mm}$. & 1,26 & $\mathrm{~mm}$ \\
\hline & 1,62 & $"$ & 1,36 & , & 0,86 & ") & 0,72 & " \\
\hline & 0,09 & , & 0,08 & n & 0,57 & n & 0,43 & ) \\
\hline & 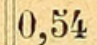 & , & 0,03 & " & 0,25 & ") & 0,18 & " \\
\hline
\end{tabular}

Es liessen sich noch andere Unterschiede anführen, z. B. die verschiedene Eigrösse; die innere Organisation jedoch scheint, soweit ich die Diplozoen miteinander verglichen habe, bei allen dieselbe zu sein. Vielleicht könnte man in diesem Falle von Wirtsvarietäten reden, welche bedingt wären durch die Grösse des Wirtes. Dann glaube ich aber, dass auch die drei Species, welche C. VoGT aufstellt, ebenfalls Varietäten sind.

Betreffs der Eierbildung des Diplozoon paradoxum v. Nordm. 
bemerkt ZELLER $(1872,1888)$, dass dieselbe bei Eintritt der kälteren Jahreszeit mitunter schon zu Ende Oktober aufhöre und erst mit der Wiederkehr der wärmeren Jahreszeit neu beginne. Meine Beobachtungen stimmen damit nicht ganz überein. Im Dezember fand ich bei meinen Exemplaren zwar auch keine Eier, dagegen besassen Diplozoen, welche frisch gefangenen Brachsen des Rheines anfangs Februar entnommen worden waren, reife Eier.

Diplozoon muss demnach auch im Stande sein während der kalten Jahreszeit Eier zur Reife zu bringen.

\section{Distomum appendiculatum Rud.}

(Apoblema Dujard.)

Fig. 11-12.

MoNTICELI.I's kritische und ausführliche Arbeit (1891) über das Genus Apoblema Duj. unterzieht auch dieses Distomum einer genauen Beschreibung. Ich füge desshalb hier nur einige kleine Bemerkungen an.

MoNTICELLI (1891) lässt den Exkretionsapparat als einfachen Stamm direkt ausmünden, im Texte führt er dazu folgendes an : "Tronco mediano del sistema escretore che si biforca dietro i testicoli in prossimità del testicolo posteriore. »

Eine Gabelung der Exkretionsgefässe findet statt. Der vereinigte Stamm führt jedoch nicht als solcher nach aussen, sondern bildet eine wohl entwickelte Exkretionsblase. An sämtlichen, von mir untersuchten Exemplaren konnte ich dieselbe mehr oder weniger deutlich sehen. Ein Bild davon habe ich in der Fig. 11 gegeben.

Der Inhalt des Sammelkanales sowie der Blase besteht aus stark lichtbrechender Substanz, wahrscheinlich Endprodukten des Stoffwechsels.

Indem ich in eine feuchte Kammer Eier des Distomum 
appendiculatum Rud. brachte, gelang es mir, dieselben zur Reife zu bringen. Ich konnte dann den aufspringenden Deckel an jedem einzelnen Ei wahrnehmen, während dies bei unreifen Eiern nicht möglich ist. Auch MonticELLI zeichnet nur ein unreifes Ei, ich habe desshalb die Zeichnung eines reifen Eies gegeben (Fig. 12).

\section{Distomum globiporum Rud.}

Finige Beobachtungen, welche ich an diesem so häufigen Distomum gemacht habe, glaube ich am besten hier kurz anführen zu können.

Von den Dotterstöcken des Distomum globiporum Rud. sagt schon v. SiEBold (1836), dass sie vier Gruppen bildeten. Looss (1894) bemerkte dass "namentlich in den vorderen und hinteren Körperteilen die Dotterstöcke besonders angehäuft sind. » Auch ZschокKE (1884) macht die Beobachtung einer Vierteilung der Dotterstöcke.

Nach meinen Beobachtungen scheint diese Gruppierung nicht durch die starke Anhäufung der Dotterstöcke, noch durch eine wirkliche, innere Gliederung entstanden zu sein, sondern ich vermute, dass sie durch die sich ausdehnenden Schlingen des Uterus herbeigeführt worden sei. Bei allen Exemplaren nämlich, wo diese Erscheinung zu Tage trat, hatte sich der Uterus von der Seite her so in die Dotterstöcke gedrängt, dass dieselben eingeschnürt wurden und nur noch schmale Verbindungsbrücken zwischen den einzelnen Teilstücken bestanden.

Looss (1894) erwähnt von den Eiern des Distomum globiporum, dass dieselben *lange vor der Vollendung ihrer Entwicklung abgelegt werden». Eier mit Embryonalzellhaufen bezeichnet er als die fortgeschrittensten Stadien, die er im Innern der Mutter antreffen konnte. Ich dagegen habe im März und Juli Distomum globiporum Rud. untersucht und gefunden, 
dass ihre Eier bei leisem Druck auf die Schale wohl entwickelte Embryonen entliessen.

Distomum globiporum Rud. ist ein charakteristischer Parasit der Cypriniden; es ist im Stande, die verschiedenen Arten dieser Fischgruppe zu lewohnen.

Die zum Schlusse angehängte Wirtstabelle mag dies am besten zeigen.

Wirte des Distomum globiporum :

1. Abramis brama.

2. . $\quad$ blicca $=$ Blicca bjorkna.

3. $\gg$ vimba.

4. Alburnus lucidus.

5. Acerina cernua.

6. Barbus fluviatilis.

7. Chondrostoma nasus.

8. Cobitis barbatula.

9. Cyprinus carpio.

10. Esox lucius.

11. Leuciscus rutilus.

12. " meidingeri.

13. " scardapha.

14. Phoxinus lavis.

15. Perca fluviatilis.

16. Scardinius erythrophthalmus.

17. Squalius cephalus.

18. 》 leuciscus.

19. Tinca vulgaris.

20. Thymallus vulgaris. 


\section{LITTERATURVERZEICHNIS}

1803. Zeder, J. G. H. - Anleitung zur Naturgeschichte der Eingeweidewürmer. Bamberg.

1823. Jurine, L. - Note sur la Douve à long cou (Fasciola lucii). Mém. de la Soc. de Phys. et d'Hist. nat. de Genève, t. II, 1 re part.

1836. v. Siebold, C. F. - Enthelminthica. Wiegmann's Archiv, I.

1841. Vogr, C. - Zur Anatomie der Parasiten. Arch. f. Anat., Phys. und wiss. Medizin von Joh. Müller, Berlin.

1845. Dujardin, F. - Histoire des Helminthes ou vers intestinaux. Paris.

1852. Wagener, G. R. - Enthelminthica. Müller's Archiv f. Anat. und Phys.

18วั7. Wagener, G. R. - Beiträge zur Entwicklungsgeschichte der Eingeweidewürmer. Natuurkundige Verhandelingen te Haarlem.

185̈8. Diesing, C. M. - Revision der Myzhelminthen. Sitzungsber. d. k. Akad. Wien, Bd. XXXII.

1858. van Beneden, J. P. - Mémoire sur les vers intestinaux. Suppl. aux Compt. rendus. Paris.

1863. Leuckart, R. - Die Parasiten des Menschen und die von ihnen herrührenden Krankheiten. Leipzig.

1872. Zeller, E. - Untersuchungen über die Entwicklung des Diplozoon paradoxum. Zeitschr. f. wiss. Zool.. Bd. XXII, Heft 2.

1877. v. Linstow, 0. - Helminthologische Notizen. Arch. f. Naturgesch. 43. Jahrgang, I.

1877. Leuthner, F. - Die mittelrheinische Fischfauna. Basel.

1882. Fatio, V. - Histoire naturelle des poissons. Faune des Vertébrés de la Suisse, vol. IV.

1883. Ziegler, H. E. - Bucephalus und Gasterostomum. Zeitschr. f. wiss. Zool., Bd. XXXIX, Heft 4.

1883. Bunge, G. - Ueber das Sauerstoffbedürfniss der Darmparasiten. Zeitschr. f. physiol. Chemie, Bd. VIII.

1884. Zsснокке, F. - Recherches sur l'organisation et la distribution zoologique des vers des poissons d'eau douce. Arch. de Biologie, t. V, Gand.

1884. Gaffron, E. - Zum Nervensystem der Trematoden. Zool. Beiträge, I. Breslau.

1886. Piesbergen, F. - Die Ekto- und Entoparasiten, von welchen die in der Umgebung von Tübingen lebenden Fische bewohnt werden. Jahreshefte des Vereins für vaterländische Naturkunde in Würltemberg, 42. Jahrg.

1886. Prenant, A. - Recherches sur les vers parasites des poissons. Bull. de la Soc. des scienc. de Nancy, série II, t. VII, fasc. XVII.

1888. Monticeld, F. S. - Contribuzioni allo studio della fauna elmintologica del golfo di Napoli. Ricerche sullo Scolex polymorphus. Mittheil. a. d. zool. Stat. Neapel, Bd. VIII. 
1888. Susta, J. - Die Ernährung des Karpfen und seiner Teichgenossen. Stettin.

1888. Zeller, E. - Ueber den Geschlechtsapparat des Diplozoon paradoxum. Zeitschr. f. wiss. Zoologie, Bd. XLVI, Heft 2.

1889. Zsснокке, F. - Erster Beitrag zur Parasitenfauna von Trutta salar. Verhandlg. der Nat. Ges. Basel. Theil VIII, Heft 3.

1890. Braun, M. - Notiz über Auswanderung von Distomen. Centralbl. f. Bakt. und Parasitenkunde, Bd. VII.

1890. Bunge, G. - Weitere Untersuchungen über die Atmung der Würmer. Zeitschr. f. physiol. Chemie, Bd. XIV.

1890. Fatio, V. - Histoire naturelle des poissons. Faune des Vertébrés de la Suisse, vol. V.

1890. v. Linstow, O. - Beitrag zur Kenntnis der Vogeltanien nebst Bemerkungen über neue und bekannte Helminthen. Arch. f. Naturgesch., Bd. LVI.

1891. Zsснокке, F. - Die Parasitenfauna von Trutta salar. Centralbl. f. Bakt. und Parasitenkunde, Bd. X.

1891. Brandes, G. - Die Familie der Holostomiden. Zool. Jahrbücher (Abtheilung für Systematik, etc.), Bd. V.

1891. Monticelli, F. S. - Osservazioni ad alcune forme del Gen. Apoblema Dujard. Atti R. Accad. sc. Torino, vol. XXVI.

1891. Seligo, A. - Die deutschen Süsswasserfische und ihre Lebensverhältnisse. Die Tier- und Pflanzenwelt des Süsswassers herausg. von Dr. 0. Zacharias, Bd. II.

1893. Braun, M. - Bronn's Klassen und Ordnungen des Tierreichs. Abt. Vermes (Trematoden).

189'. Looss, A. - Die Distomen unserer Fische und Frösche. Bibliotheca zoologica, Bd. VI, Heft 16.

1896. Hausmann, L. - Ueber Trematoden der Süsswasserfische (Vorläufige Mitteilung). Centralbl. f. Bakt., Parasitenkunde und Infektionskrankheiten, Bd. XIX. 


\section{FIGURENERKLERUNG}

Alle Umrisse der Zeichnungen sind mit dem Abbé'schen Zeichnungsapparat entworfen worden.

Für alle Figuren gelten folgende Bezeichnungen :

$$
\begin{aligned}
M s & =\text { Mundsaugnapf. } \\
V & =\text { Vorhof. } \\
P h & =\text { Pharynx. } \\
O e & =\text { Oesophagus. } \\
D & =\text { Darm. } \\
B s & =\text { Bauchsaugnapf. } \\
H & =\text { Hoden. } \\
V e & =\text { Vasa efferentia. } \\
V d & =\text { Vas deferens. } \\
V s & =\text { Vesicula seminalis. } \\
C i & =\text { Cirrusbeutel. } \\
K & =\text { Keimstock. } \\
K g & =\text { Keimgang. } \\
L C & =\text { Laurerscher Canal. } \\
R s & =\text { Receptaculum seminis. } \\
D s t & =\text { Dotterstöcke. } \\
D g & =\text { Dottergang. } \\
U & =\text { Uterus. } \\
E x & =\text { Exkretionsstamm. } \\
E x x_{1} & =\text { Exkretionsnebenslämme. } \\
E x b & =\text { Exkretionsblase. } \\
E x p & =\text { Exkretionsporus. }
\end{aligned}
$$

Fig. 1. Distomum angusticolle n. sp. N, Nerv; $Z$, grosse Zellen des Mundsaugnapfes und des Pharynx.

Fig. 2. Distomum angusticolle n. sp. Längsschnitt durch die Darmwand; Lm, Längsmuskeln; Rm, Ringmuskeln ; $E$, Epithel .

Fig. 3. Distomum angusticolle $\mathrm{n} . \mathrm{sp}$. Ei.

Fig. 4. Distomum exspinosum n. var. Gp, Genitalporus; Dej, Ductus ejaculatorius ; $\mathrm{Va}$, Vagina ; $\mathrm{Dr}$, Dotterreservoir.

Fig. こ. Distomum exspinosum n. var. Ei.

Fig. 6. Gasterostomum fimbriatum v. Siebold. Microphotographische Aufnahme.

Fig. 7. Gasterostomum fimbriatum v. Siebold. Microphotographische Auf. nahme des Kopfsaugnapfes im Ruhezustand.

Fig. 8. Gasterostomum fimbriatum v. Siebold. Kopfsaugnapf ; $T$, grosser T'entakel, $T_{2}$, kleiner Tentakel.

Fig. 9. Tetracotyle spec.? Microphotographische Aufnahme.

Fig. 10. Tetracotyle spec.? in anderer Lage.

Fig. 11. Distomum appendiculatum Rud. Hinteres Körperende mit eingezogenem Schwanzteile.

Fig. 12. Distomum appendiculatum Rud. Reifes Ei. 

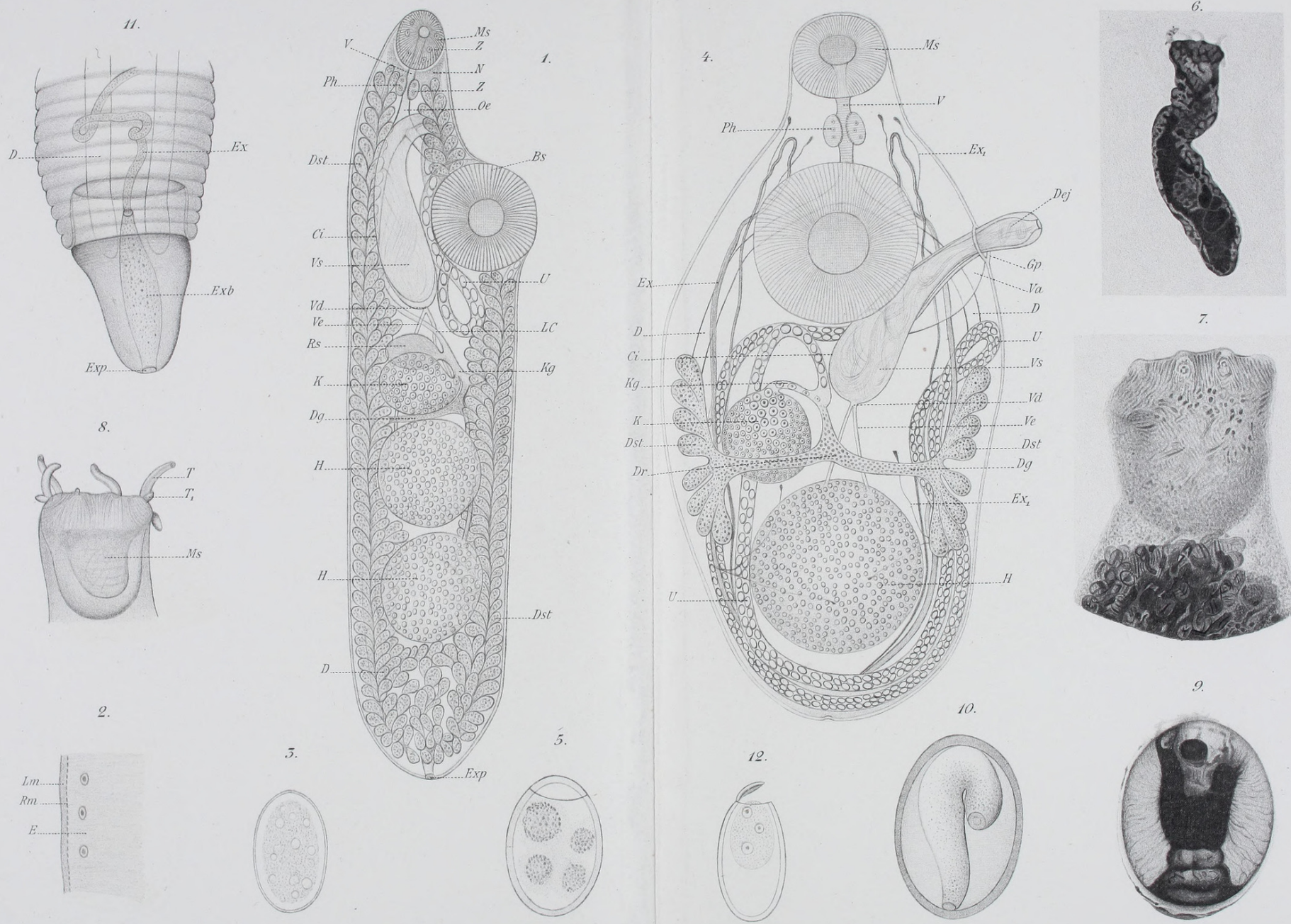


\section{$2 \mathrm{BHL}$ Biodiversity Heritage Library}

Hausmann, Leopold. 1897. "Ueber Trematoden der Süsswasserfische." Revue suisse de zoologie 5(1), 1-42. https://doi.org/10.5962/bhl.part.49548.

View This Item Online: https://www.biodiversitylibrary.org/item/110849

DOI: https://doi.org/10.5962/bhl.part.49548

Permalink: https://www.biodiversitylibrary.org/partpdf/49548

\section{Holding Institution}

Harvard University, Museum of Comparative Zoology, Ernst Mayr Library

\section{Sponsored by}

Harvard University, Museum of Comparative Zoology, Ernst Mayr Library

\section{Copyright \& Reuse}

Copyright Status: Public domain. The BHL considers that this work is no longer under copyright protection.

This document was created from content at the Biodiversity Heritage Library, the world's largest open access digital library for biodiversity literature and archives. Visit BHL at https://www.biodiversitylibrary.org. 